\title{
Auditory Processing Performance and Nonsensory Factors in Children with Specific Language Impairment or Auditory Processing Disorder
}

\author{
Melanie A. Ferguson, MSc. ${ }^{1}$ and David R. Moore, Ph.D. ${ }^{1,2,3}$
}

The nature of auditory processing deficits is at the heart of understanding auditory processing disorder (APD) in children. This article reviews evidence that confounding nonsensory factors, including maturation, processing efficiency, and cognition, influence auditory processing in children with APD or language learning impairment. Experimental evidence is presented to show that performance thresholds on nonspeech auditory processing (AP) tests, such as tone detection tasks in noise or in quiet, are poorer in children clinically diagnosed with APD or specific language impairment (SLI), compared to typically developing mainstream school (MS) children. However, with the exception of backward masking, these group differences disappeared for all AP tests after accounting for nonverbal IQ. Intrinsic attention, indexed by variability in AP test performance, was examined alongside AP threshold performance. Generally there was no difference in intrinsic attention across the three participant groups (MS, SLI, APD), but frequency discrimination (FD) was an exception. Thus, although reduced intrinsic attention has been shown to be a factor in the presenting symptoms of APD, including poor listening, speech intelligibility, and communication, there is, surprisingly, no robust evidence that intrinsic attention in children identified with APD or SLI is any poorer than that in typically developing children.

KEYWORDS: Auditory processing, cognition, attention, language, developmental disorder

${ }^{1}$ NIHR Nottingham Hearing Biomedical Research Unit, Nottingham University Hospitals, NHS Trust; ${ }^{2} \mathrm{MRC}$ Institute of Hearing Research, Nottingham, United Kingdom; ${ }^{3}$ Cincinnati Children's Hospital, Cincinnati, Ohio.

Address for correspondence: Melanie Ferguson, MSc., NIHR Nottingham Hearing Biomedical Research Unit, 113 The Ropewalk, Nottingham, United Kingdom, NG1 5DU (e-mail: melanie.ferguson@nottingham.ac.uk).
New Evidence in Auditory Processing Research; Guest Editor, Deborah Moncrieff, Ph.D.

Semin Hear 2014;35:1-14. Copyright (C) 2014 by Thieme Medical Publishers, Inc., 333 Seventh Avenue, New York, NY 10001, USA. Tel: +1(212) 584-4662.

DOI: http://dx.doi.org/10.1055/s-0033-1363520.

ISSN 0734-0451. 
Learning Outcomes: As a result of this activity, the participant will be able to (1) describe how nonsensory factors influence auditory processing thresholds and (2) explain the difference in auditory processing performance (thresholds and intrinsic attention) between typically developing children and those with auditory processing disorder or specific language impairment.

Deficits in auditory perception, or "processing," are central to definitions of auditory processing disorder (APD). ${ }^{1}$ In the position statement from the British Society of Audiolo$\mathrm{gy},{ }^{2}$ these deficits are identified specifically for both speech and nonspeech sounds. The development of the previous British Society of Audiology definition in $2007^{3}$ (see footnote ${ }^{*}$ ) to the current $2011^{2}$ position statement was primarily informed by a large UK population study of normally hearing mainstream school (MS) children age 6 to 11 years. ${ }^{4}$ This study tested, and then rejected, the hypothesis that APD resulted from impaired sensory (temporal or frequency) processing skills. Furthermore, it concluded that the presenting symptoms of APD, namely difficulties in listening, speech in noise intelligibility, and communication, were not related to auditory processing sensory deficits. Instead, these functional difficulties were best predicted by the children's response variability in performing auditory processing tasks (intrinsic attention) and by other reduced cognitive abilities. In short, the deficits were "perceptual" rather than "sensory," where perception means the "organization, identification, and interpretation of sensory information." These conclusions from Moore et al, ${ }^{4}$ specific to APD, are consistent with a wider body of evidence that disputes the hypothesis that deficits in auditory sensory processing cause language learning impairments (LLIs). ${ }^{6-10}$

Much of the research on the role of auditory processing has focused on LLI (e.g., specific language impairment [SLI] and dyslexia), which, although heterogeneous in nature, have been suggested as being better specified than

\footnotetext{
$B S A(2007)^{3}$ definition of APD states " $A P D$ results from impaired neural function and is characterized by poor recognition, discrimination, separation, grouping, localization, or ordering of non-speech sounds. It does not result from a deficit in general attention, language or other cognitive processes."
}

APD. ${ }^{11}$ The early findings of Tallal and Piercy led to the proposal that LLI is caused by temporal auditory deficits, specifically relating to short duration or rapidly fluctuating sounds. ${ }^{12}$ According to this proposal, poor auditory temporal perception causes poor speech (phonological) perception, which then impacts on language acquisition and reading. A role for impaired temporal processing was further supported by evidence including deficits in backward masking (BM) in children with SLI, ${ }^{13}$ and in frequency modulation (FM) detection $^{14,15}$ and tone repetition ${ }^{16}$ in children with dyslexia. However, numerous studies have shown that although auditory processing deficits, including both temporal and spectral deficits, did occur in children with LLI, ${ }^{17,18}$ they were usually present in only a minority of cases. ${ }^{6,19,20}$ Furthermore, there was usually a substantial overlap in auditory processing ability between children with LLI and typically developing children. ${ }^{7,9,21-23}$

Suggestions that auditory deficits are due to nonsensory factors, including greater "internal noise,"24,25 maturation, ${ }^{4,26,27}$ and attention, ${ }^{28-31}$ rather than to sensory factors, have also been gaining momentum over the last decade. For example, normal "processing efficiency" in hearing is attributable in part to compressive nonlinearity of the basilar membrane. ${ }^{32}$ A processing efficiency model, based on this normal function of the cochlea rather than on impaired sensory processing, can explain why performance on (temporal) BM tasks is apparently poorer than performance on (nontemporal) simultaneous masking. Auditory processing tasks also have been shown to have different developmental trajectories during normal maturation, ${ }^{4,26,33}$ potentially leading to inappropriate conclusions about delayed development of temporal processing. For example, Moore et al showed that maturational improvements in frequency discrimination (FD) thresholds continued to improve into adulthood, 
whereas thresholds from (temporal) BM were mature by 10 to 11 years, and other (nontemporal) tone detection in noise tasks, such as simultaneous masking, were fully developed by around 8 to 9 years. ${ }^{33}$

The role of attention in auditory task performance has been gaining momentum since greater variability in task performance was originally suggested to be associated with lapses in attention. ${ }^{34}$ Furthermore, task response variability is more likely to be evident in clinical groups, a result being that cases with extreme variability in performance can have a disproportionately negative effect on the group mean. ${ }^{35}$ The role of attention has been followed up more recently in both typically developing children ${ }^{4,26,36}$ and in children with attention deficit hyperactivity disorder (ADHD). ${ }^{31}$ In typically developing children, between-individual threshold variability is to be greater in younger age groups, particularly for those tasks that show longer maturational effects. ${ }^{26,33}$

Within-individual variability can be indexed by several different measures, for example, the standard deviation of reversals within a track, the standard deviation of each trial value within a track, or the threshold difference across two tracks. It has been proposed that withinindividual measures of response variability provide an index of intrinsic attention, ${ }^{4,26,31,36}$ in that the attention metric is incorporated within the auditory task. This is contrasted with extrinsic attention tasks, which are more typical, stand-alone measures of attention that clearly involve complex and supramodal processing (e.g., the Test of Everyday Attention for Children $^{37}$ ). In a study of children with ADHD who performed frequency discrimination and FM detection tasks while on and off stimulant medication to control hyperactivity and inattention, intrinsic attention improved only for frequency discrimination. ${ }^{31}$ Taken together, the studies reported here suggest that some auditory tasks may be more affected than others by factors related to age and attention.

But how does poor intrinsic attention affect everyday listening abilities of children? There have been no reports of this in children who have been diagnosed with APD per se. However, Moore et al (2010) found that intrinsic attention and cognition were the main predictors of the typical presenting symptoms of APD. ${ }^{4}$ These symptoms included parental report of listening and communication as indicated by the CHAPPS (Children's Auditory Processing Performance Scale $)^{38}$ and the CCC2 (Children's Communication Checklist-2) ${ }^{39}$ questionnaires, respectively, and speech intelligibility, as indicated by a VCV (vowel-consonant-vowel) nonsense syllable-in-noise task. Sensory processing, as evidenced by derived temporal and spectral resolution thresholds, accounted for very little of the variance in these presenting symptoms. Thus, it was proposed that APD is primarily a cognitive (e.g., attention) disorder rather than a specific auditory sensory processing disorder. ${ }^{4}$

This recent evidence that cognition plays an underlying role in listening difficulties in children, whether diagnosed as APD or LLI, is not new. Associations between auditory perceptual performance and intelligence were reported in the $1990 \mathrm{~s}^{40,41}$ and, indeed, date back to Spearman in $1904 .{ }^{42}$ However, the effect of cognition on auditory processing (and also visual processing) was often not measured in some of the earlier studies in children with LLI. In part, this was because the working definition of LLI required that nonverbal IQ (NVIQ) levels were normal, and a common study exclusion criterion was that NVIQ (also known as "performance IQ" or "fluid intelligence") was below normal levels. ${ }^{43,44}$ The same was also true in adults with dyslexia., ${ }^{8,45}$ Although these studies showed significant effects of auditory and visual perceptual processing on reading, a reanalysis of the data showed that the variance of auditory and visual perceptual tasks that accounted for reading abilities was significantly reduced after taking NVIQ into account. ${ }^{46}$ Thus, NVIQ was implicated as an integral factor in the performance of perceptual processing tasks. As NVIQ is also closely interrelated with other cognitive processes (e.g., memory and attention), then it is likely that these processes also may affect performance on simple or complex perceptual tasks.

This conclusion was generally supported in later studies where NVIQ and other measures of cognitive performance were not exclusion criteria. A study of children with dyslexia 
showed that, after accounting for NVIQ, $2-\mathrm{Hz}$ FM detection thresholds retained some, albeit a reduced, relation with reading. ${ }^{14}$ Another study of children and young adults with a wide range of full-scale IQ levels showed that auditory (2-Hz: FM and AM) and visual (coherent motion detection) tasks were no longer significantly related to word reading after controlling for IQ. ${ }^{47}$ Furthermore, NVIQ, verbal IQ, and memory were shown to be significantly poorer in children identified with APD or SLI compared with typically developing children, with no significant differences between the APD or SLI groups. ${ }^{48,49}$ A study of teenagers with a grammatical version of SLI demonstrated a strong link between NVIQ and language.' However, in children suspected of having APD, there was no evidence to suggest a direct association between cognitive and auditory performance, despite lower cognitive and auditory sensory processing abilities in the suspected APD group compared to typically developing children. ${ }^{50}$ Cognition is now widely recognized as playing an important role in listening and hearing in those with developmental disorders and other special populations, notably older adults. ${ }^{51-53}$

The aims of the research reported here were to assess auditory processing performance in three groups of children (MS, SLI, and APD) in terms of (1) auditory processing threshold ${ }^{\ddagger}$ and (2) intrinsic attention indexed by response variability. Based on the work of Moore et al, ${ }^{4}$ three hypotheses were examined: that the two clinical groups (SLI and APD) would underperform on both (1) auditory processing test thresholds and (2) response variability measures, but (3) there would be no difference across the three groups on the derived threshold measures, as nonsensory factors including attention would be subtracted out. ${ }^{4,54}$

\section{METHODS}

\section{Participants}

Participants age 6 to 13 years were recruited through two separate studies ${ }^{33,49}$ and were

\# Results on AP thresholds from the MS children have been reported elsewhere. ${ }^{33}$ included if they met the general inclusion criteria of (1) normal air-conduction thresholds $(\leq 20-\mathrm{dB}$ hearing level (HL) at $0.5,1,2$, and $4 \mathrm{kHz}$ ), (2) normal middle ear function (middle ear pressure $\geq-150 \mathrm{daPa}$ and compliance $\geq 0.2 \mathrm{cc}$ ), and (3) English as the main home language. The first study included 75 children from MS (mean age $=8.4$ years, standard deviation $[\mathrm{SD}]=1.6 ; 36$ girls, 39 boys). ${ }^{33}$ The second study included 88 children, including those from MS $(n=47$; mean age $=8.6$ years, $\mathrm{SD}=2.0 ; 21$ girls, 26 boys) and those who received a clinical diagnosis of SLI $(n=22 ;$ mean age $=8.4$ years, $\mathrm{SD}=1.6 ; 8$ girls, 14 boys $)$ or APD ( $n=19$; mean age $=9.7$ years $\mathrm{SD}=1.8 ; 6$ girls, 13 boys). ${ }^{49}$

MS children were those who attended mainstream schools and who were not specifically screened for developmental disorders. Children with SLI were identified and recruited through the local Speech and Language service if they fulfilled the clinical criteria for SLI. The criteria were based on Leonard's "diagnosis by exclusion" such that they had significant speech or language difficulties that could not be accounted for by factors including hearing loss, autism, learning or physical disability, or dual language background. ${ }^{55}$ This diagnostic approach is one that is widely used across many UK Speech and Language services.

Children with APD were recruited through the local audiology or ear, nose, and throat service. They were audiometrically normal and had been identified as having one or more symptoms of APD, specifically difficulties in the following: hearing in background noise (68.4\%), staying focused or being easily distracted (57.8\%), remembering complex and multistep instructions (52.6\%), understanding when listening (21.0\%), and expressing or clearly using speech (10.5\%). There are a host of well-recognized issues around the diagnosis of APD, including the lack of a "gold standard," $49,56-58$ disputes over diagnostic criteria, ${ }^{58-60}$ use of poorly specified or validated diagnostic tests, ${ }^{49,57,61}$ and lack of difference between presenting symptoms in those diagnosed with APD and those without APD. ${ }^{62}$ Due to the wide disparity in diagnostic approaches to APD in the UK, ${ }^{63}$ this selection approach was consistent with the UK-wide 
accepted practice at that time of using presenting symptoms to diagnose APD based on those reported in the literature. ${ }^{64,65}$

\section{Test Procedures}

\section{AUDIOLOGICAL MEASURES}

Pure tone air-conduction thresholds were obtained for each ear at $0.25,0.5,1,2,4$, and $8 \mathrm{kHz}$ using a Siemens Unity audiometer and TDH-49P headphones (Telephonics, New York, NY) in a sound-attenuating booth. Middle ear function was assessed by otoscopy, tympanometry, and acoustic reflex thresholds using a GSI Tympstar (Grason-Stadler, Eden Prairie, MN).

\section{AUDITORY PROCESSING TESTS}

A schematic representation of the tests is summarized in Fig. 1. Temporal integration thresholds were obtained from the difference between two individual $1000-\mathrm{Hz}$ tone detection-in-quiet tasks with tone duration 20 milliseconds $(1 \mathrm{k} 20)$ and 200 milliseconds $(1 \mathrm{k} 200)$. Interstimulus intervals were 700 milliseconds and 500 milliseconds, and initial intensities were $80-$ and $60-\mathrm{dB}$ sound pressure level (SPL) for $1 \mathrm{k} 20$ and $1 \mathrm{k} 200$, respectively. Frequency resolution thresholds were obtained from the difference between two $1000-\mathrm{Hz}$ tone-detection-

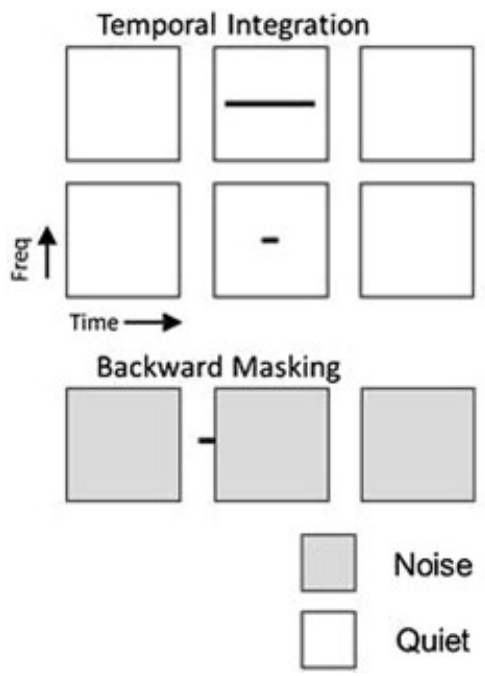

in-simultaneous-masking noise tasks. The nonotch condition (SM0) was a bandpass noise (600-Hz to $1400-\mathrm{Hz}$, center frequency $1000-$ $\mathrm{Hz}$ ), and the notch condition (SMN) was a spectrally notched noise $(400-\mathrm{Hz}$ notch, centered on $1000-\mathrm{Hz}$ within a $400-\mathrm{Hz}$ to $1600-\mathrm{Hz}$ noise band). Noise duration was 300 milliseconds and noise level was 40-dB SPL. The 20-millisecond tone was presented 200-milliseconds after onset of the noise. Interstimulus intervals were 400-milliseconds and initial tone intensities were 85- and 70-dB SPL for SM0 and SMN, respectively. BM thresholds were obtained for a $1000-\mathrm{Hz}, 20$-millisecond tone, presented immediately ( 0 -millisecond gap) prior to a bandpass noise (same as for SM0). Initial intensity was 90-dB SPL. Frequency discrimination thresholds were obtained from a standard 200-millisecond tone fixed at $1000-\mathrm{Hz}$ and a target 200-millisecond tone adjusted adaptively toward the standard from an initial frequency of $1500-\mathrm{Hz}$ (i.e., standard plus 50\%). The interstimulus interval was 400 milliseconds and intensity was fixed at 70-dB SPL. All tones had a 10-millisecond cosine-squared ramp. A familiarization track, described elsewhere, ${ }^{33}$ preceded each auditory processing test.

The stimuli were generated using IHRSTAR software running a three-interval, threealternative forced choice, "oddball" response paradigm and delivered through Sennheiser
Frequency resolution

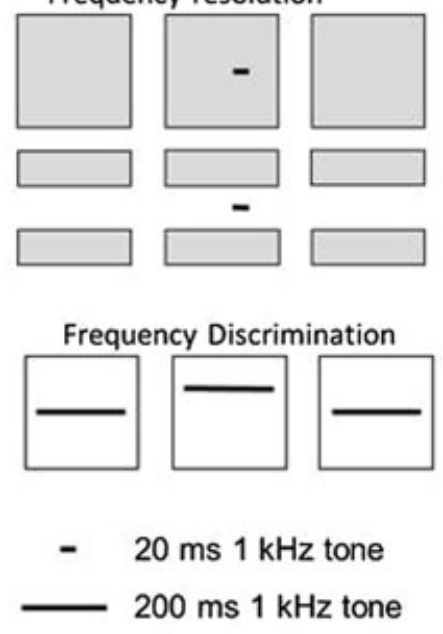

Figure 1 Schematic representing the stimulus parameters for the auditory processing tasks. 
HD 25-1 headphones (Sennheiser, Hanover, Germany). Stimuli were adjusted adaptively using a three-phase adaptive staircase procedure with an initial one-down, one-up rule, followed by a three-down, one-up rule. ${ }^{66}$ For the detection tests, the initial step size was $10-\mathrm{dB}$, reduced to $5-$ and $3-\mathrm{dB}$ over the next two reversals. The frequency discrimination test used an initial multiplicative step size of 2, changing to square root of 2 (1.412) after the first two reversals. Two tracks were obtained for all tests, and a third was obtained if a discrepancy criterion was exceeded. The track threshold was the averaged stimulus level of the last two reversals. For the discrimination tasks, the geometric mean was obtained. The overall threshold for each task was the average of the two track thresholds (geometric mean for frequency discrimination). Where a third track was obtained, the overall threshold was that from the two tracks that had the closest thresholds.

The variability of the responses within phase 3 was captured from the first two tracks of each test by two measures (1) the unsigned intertrack threshold difference (ITTD), ${ }^{36}$ and (2) the mean $\mathrm{SD}$ of the data points in phase 3 for each of the two tracks (geometric SD for discriminations tasks), which were averaged across both tracks to give an overall SD score. These two measures were used to index intrinsic attention.

Individual measures were defined as the performance thresholds of each discrete, standalone test (the six tests in Fig. 1). Such thresholds are determined by both sensory and nonsensory (e.g., cognition and fatigue) factors. ${ }^{4,33}$ Derived measures were the difference in thresholds between two individual tests (frequency resolution, temporal integration). This subtraction removed many nonsensory factors that are consistent for an individual participant, thus providing a measure of sensory performance. ${ }^{4,54}$

\section{NONVERBAL IO}

The Matrix Reasoning subtest of the Wechsler Abbreviated Scale of Intelligence was used to obtain measures of nonverbal IQ. ${ }^{67}$ This subtest is a measure of general intelligence and nonverbal fluid reasoning, and scores for each subtest were standardized in accordance with ageequivalent norms.

\section{SESSIONAL PROCEDURE}

Participants typically attended two test sessions, each approximately 2 hours in duration, with at least one break per session. Auditory processing tasks were interleaved with cognitive and speech tests (for results see Ferguson et $\mathrm{al}^{49}$ ) to provide a varied test structure and to maintain motivation and alertness. Participants were tested in a double-walled, sound-attenuating booth.

\section{Statistical Analysis}

Distribution for auditory processing thresholds, within-track SD measures and auditory processing ITTD (unsigned) were highly skewed and Kolmogorov-Smirnov tests for normality were significant $(p<0.05)$. Thresholds were $\log$-transformed to return normal distributions. As some of the ITTD data points were 0, 0.5 was added to the raw ITTD data prior to the log-transformation, which resulted in normal distributions. To minimize the effects of multiple comparisons that may lead to type I errors, multivariate analysis of variance (MANOVA) was performed where necessary. Auditory processing tasks were grouped and analyzed as either tone detection tasks for the individual measures (i.e., $1 \mathrm{k} 200,1 \mathrm{k} 20$, BM, SM0, SMN) or derived measures (i.e., TR, frequency resolution). Where there were significant effects (Wilks Lamda, $\lambda<0.05$ ), post hoc testing was performed using univariate analyses of variance and pairwise comparisons. Further correction for multiple comparisons (e.g., Bonferroni) was not necessary. Frequency discrimination was analyzed separately and was not included in the MANOVA because the nature of the measure was qualitatively different to that of the detection tasks. Significance level was set to $p \leq 0.05$.

\section{RESULTS}

Among the MS children, there was a significant effect of age on auditory processing thresholds for all tests $(p<0.001)$ where the youngest ( 6 to 7 years) children had poorer auditory processing thresholds than the oldest (10 to 11 years) children (see Moore et $\mathrm{al}^{33}$ for more details). Similar results were seen for both response 
variability measures $(p<0.05)$, with the exception of BM and SM0 for the SD measure. Consequently, the threshold, ITTD, and SD data for all participants, including the clinical groups, were age-standardized, based on data from the MS group, after excluding outliers who performed greater than the mean $+2 \mathrm{SD}$.

Box plots of the auditory processing threshold (z-scores) for the three groups (MS, SLI, APD) showed that the SLI and APD groups generally had poorer thresholds than the MS group (Fig. 2). MANOVA showed a significant main effect of group on auditory processing thresholds for all the individual detection measures, except SM0 (Table 1). Post hoc pairwise testing showed that the SLI and APD groups had significantly higher (poorer) thresholds than the MS group for all the individual tasks including frequency discrimination. There was no difference in performance between the APD and SLI groups. For the derived measures, there was no overall difference between groups for frequency resolution thresholds. Temporal integration times were longer for the SLI group only compared with the MS group.

Poor auditory processing performers were identified as those with $\mathrm{z}$-scores greater than 1.64. This cutoff was chosen as equivalent to the poorest $5 \%$ in a typical population to reflect published estimates of the prevalence of APD. ${ }^{68,69}$ Table 2 shows that the overall percentage of poor performers in the MS group was close to that expected (5\%). For the clinical groups, the percentage of poor performers for the individual tests ranged between 10 and $45 \%$. Where the percentage of poor performers for any individual test was $13.9 \%$ in the MS group, this was considerably higher at 54.5 and $52.5 \%$ for the SLI and APD groups, respectively. As there was no significant difference between mean performance of the SLI and APD groups for any test (Table 1), both groups were collapsed into one for statistical comparison in Table 2. The combined SLI/APD group contained significantly more children $\left(\boldsymbol{x}^{2}\right)$ who were poor performers than the MS group for most of the tests.
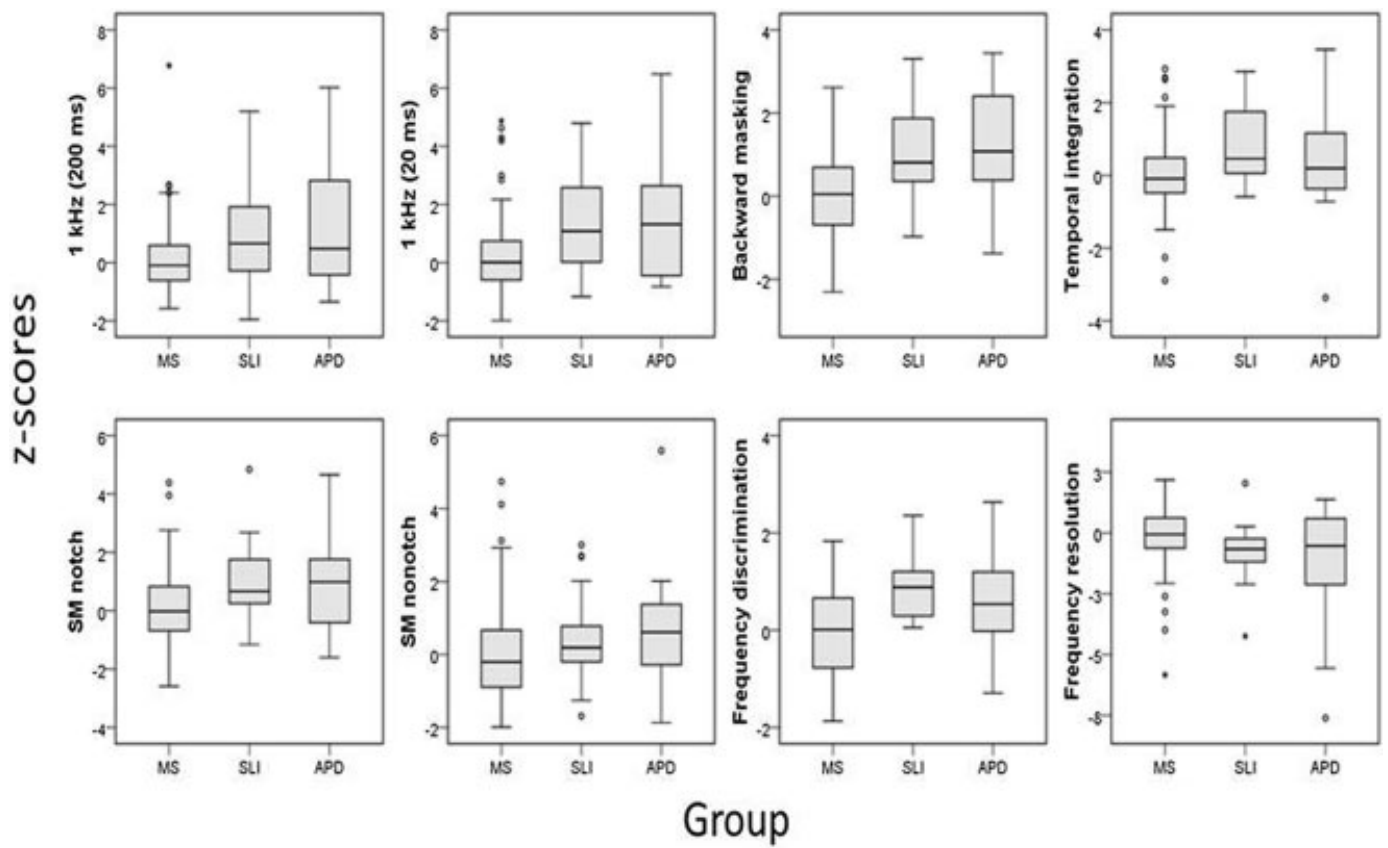

Figure 2 Box plots (median \pm interquartile range), showing the age-standardized z-scores for auditory processing thresholds (log-transformed) between the mainstream school (MS; $n=47$ ), specific language impairment (SLI; $n=21$ ), and auditory processing disorder (APD; $n=19$ ) groups. The whiskers represent the range, outliers: ${ }^{\circ} \geq 1.5$ times the interquartile range, ${ }^{*} \geq 3$ times the interquartile range. Abbreviation: SM, simultaneous masking. 
Table 1 MANOVA of the Age-Standardized z-Scores for AP Threshold by Clinical Group (MS, SLI, APD)

\begin{tabular}{|c|c|c|c|c|c|c|}
\hline \multirow[t]{2}{*}{ Test } & \multicolumn{3}{|c|}{ MANOVA Overall } & \multicolumn{3}{|c|}{ Pairwise Tests $(p)$} \\
\hline & $\overline{\text { df }}$ & $\mathbf{F}$ & $p$ & MS vs. SLI & MS vs. APD & $\overline{\text { SLI vs. APD }}$ \\
\hline AP detection & 10,188 & 2.9 & 0.002 & 0.002 & 0.006 & NS \\
\hline $1 k 200$ & 2,106 & 5.3 & 0.006 & 0.02 & 0.009 & NS \\
\hline $1 \mathrm{k} 20$ & 2,108 & 4.8 & 0.01 & 0.014 & 0.024 & NS \\
\hline BM & 2,108 & 11.5 & $<0.001$ & 0.001 & $<0.001$ & NS \\
\hline SMO & 2,111 & 1.6 & NS & - & - & - \\
\hline SMN & 2,107 & 5.1 & 0.008 & 0.016 & 0.014 & NS \\
\hline FD & 2,78 & 6.8 & 0.002 & 0.001 & 0.020 & NS \\
\hline Derived AP & 4,188 & 2.8 & 0.026 & 0.002 & NS & NS \\
\hline TI & 2,108 & 4.3 & 0.016 & 0.006 & NS & NS \\
\hline FR & 2,100 & 2.6 & NS & - & - & - \\
\hline
\end{tabular}

Post hoc ANOVA and pairwise tests are shown. Empty cells indicate where the ANOVA was not significant. 1k20, tone duration 20 milliseconds; $1 \mathrm{k} 200$, tone duration 200 milliseconds; ANOVA, analysis of variance; AP, auditory processing; APD, auditory processing disorder; BM, backward masking; FD, frequency discrimination; FR, frequency resolution; MANOVA, multivariate analysis of variance; MS, mainstream school; NS, not significant; SLI, specific language impairment; SM0, no-notch condition; SMN, notch condition; TI, temporal integration.

Although there was no significant relationship between NVIQ and auditory processing thresholds for the MS group, for the clinical groups NVIQ was significantly correlated with $\mathrm{BM}$ and FD. Across all three groups, NVIQ was significantly correlated with auditory processing threshold on all the individual tests and with temporal integration (Table 3). Consequently, the analysis in Table 1 was repeated with NVIQ as a covariate. MANOVA showed a borderline main effect of participant group for both the individual $(\mathrm{F}(10,184)=1.83$, $p=0.057)$ and the derived measures $(\mathrm{F}(4$,
$184)=2.32, p=0.058)$. There was no significant difference between the groups on frequency discrimination after accounting for NVIQ ( F $(2,78)=1.78, p=0.17)$. Among the other individual tests, post hoc analysis showed that only thresholds for BM differed between the groups $(\mathrm{F}(3,107)=5.62, p=0.007)$. The MS group performed significantly better than both the SLI and APD groups for BM $(p<0.05)$, with no difference between the SLI and APD groups.

Response variability for the three groups (MS, SLI, APD) is shown in Fig. 3. For the

Table 2 The Number and Percentage of Each Group That Exceeded a z-Score of 1.64, Equivalent to the Bottom $5 \%$ of a Normal (Typical) Population

\begin{tabular}{|c|c|c|c|c|c|c|c|}
\hline \multirow[t]{2}{*}{$\overline{\text { Test }}$} & \multicolumn{2}{|c|}{ MS $(n=47)$} & \multicolumn{2}{|c|}{ SLI $(n=21)$} & \multicolumn{2}{|c|}{ APD $(n=19)$} & \multirow{2}{*}{$p^{\chi^{2}}$} \\
\hline & $\overline{\mathrm{n}}$ & $\%$ & $\overline{\mathbf{n}}$ & $\%$ & $\overline{\mathbf{n}}$ & $\%$ & \\
\hline $1 k 200$ & 6 & 8.2 & 5 & 25.0 & 8 & 44.4 & 0.001 \\
\hline 1 k20 & 8 & 10.9 & 9 & 45.0 & 6 & 33.3 & 0.001 \\
\hline BM & 4 & 5.5 & 6 & 32.5 & 8 & 44.4 & $<0.001$ \\
\hline SMO & 8 & 10.8 & 4 & 21.1 & 2 & 10.5 & NS \\
\hline SMN & 4 & 5.6 & 5 & 27.8 & 5 & 27.8 & 0.002 \\
\hline FD & 3 & 6.9 & 2 & 10.5 & 4 & 23.5 & NS \\
\hline TI & 5 & 6.9 & 6 & 31.6 & 2 & 11.1 & 0.03 \\
\hline $\mathrm{FR}$ & 2 & 2.9 & 1 & 5.9 & 0 & 0.0 & NS \\
\hline
\end{tabular}

The $\chi^{2}$ significance level is the comparison between the MS and combined SLI/APD groups. For FR, the criterion zscore was -1.64. $1 \mathrm{k} 20$, tone duration 20 milliseconds; $1 \mathrm{k} 200$, tone duration 200 milliseconds; APD, auditory processing disorder; BM, backward masking; FD, frequency discrimination; FR, frequency resolution; MS, mainstream school; NS, not significant; SLI, specific language impairment; SM0, no-notch condition; SMN, notch condition; TI, temporal integration. 
Table 3 Correlation Coefficients between Age-Standardized Scores of AP Thresholds and Nonverbal IQ for the MS, Combined SLI/ APD Group, and the Whole Sample

\begin{tabular}{llll}
\hline Test & MS & SLI/APD & All Children \\
\hline $1 \mathrm{k} 200$ & -0.22 & -0.06 & $-0.27^{\dagger}$ \\
$1 \mathrm{k} 20$ & -0.17 & -0.29 & $-0.35^{\ddagger}$ \\
BM & -0.03 & $-0.33^{*}$ & $-0.34^{\ddagger}$ \\
SM0 & -0.25 & -0.30 & $-0.31^{\ddagger}$ \\
SMN & -0.10 & -0.19 & $-0.26^{\dagger}$ \\
FD & -0.24 & $-0.45^{\dagger}$ & $-0.46^{\ddagger}$ \\
TI & -0.81 & -0.29 & $-0.23^{*}$ \\
FR & -0.16 & -0.12 & -0.09 \\
\hline
\end{tabular}

$1 \mathrm{k} 20$, tone duration 20 milliseconds; $1 \mathrm{k} 200$, tone duration 200 milliseconds; ANOVA, analysis of variance; AP. auditory processing; $A P D$, auditory processing disorder; $B M$, backward masking; FD, frequency discrimination; $\mathrm{FR}$, frequency resolution; MANOVA, multivariate analysis of variance; MS, mainstream school; NS, not significant; SLI, specific language impairment; SM0, no-notch condition; SMN, notch condition; TI, temporal integration.

${ }^{*} p \leq 0.05$

${ }^{+} p \leq 0.01$

${ }^{\ddagger} p \leq 0.001$. detection tasks, SD measures were generally higher for the clinical groups compared with the MS group, but there was no significant overall effect of group $(\mathrm{F}(10,194)=1.14, p=0.34)$. There was also no significant overall effect of group on $\operatorname{ITTD}(\mathrm{F}(10,182)=.60, p=0.81)$. These results suggest that the group means for intrinsic attention, as indicated by within-response variability for detection tasks, do not differ between the three participant groups. For frequency discrimination, there was a significant effect of group for $\operatorname{ITTD}(\mathrm{F}(2,78)=4.9$, $p=0.009)$ but not for $\mathrm{SD}(\mathrm{F}(2,76)=1.12$, $p=0.56)$. Post hoc testing for the frequency discrimination ITTD measure showed significantly more variability for the SLI group compared with the MS group only $(p=0.004)$. There was a nonsignificant, although borderline poorer performance for the APD group compared with the MS group $(p=0.06)$ and
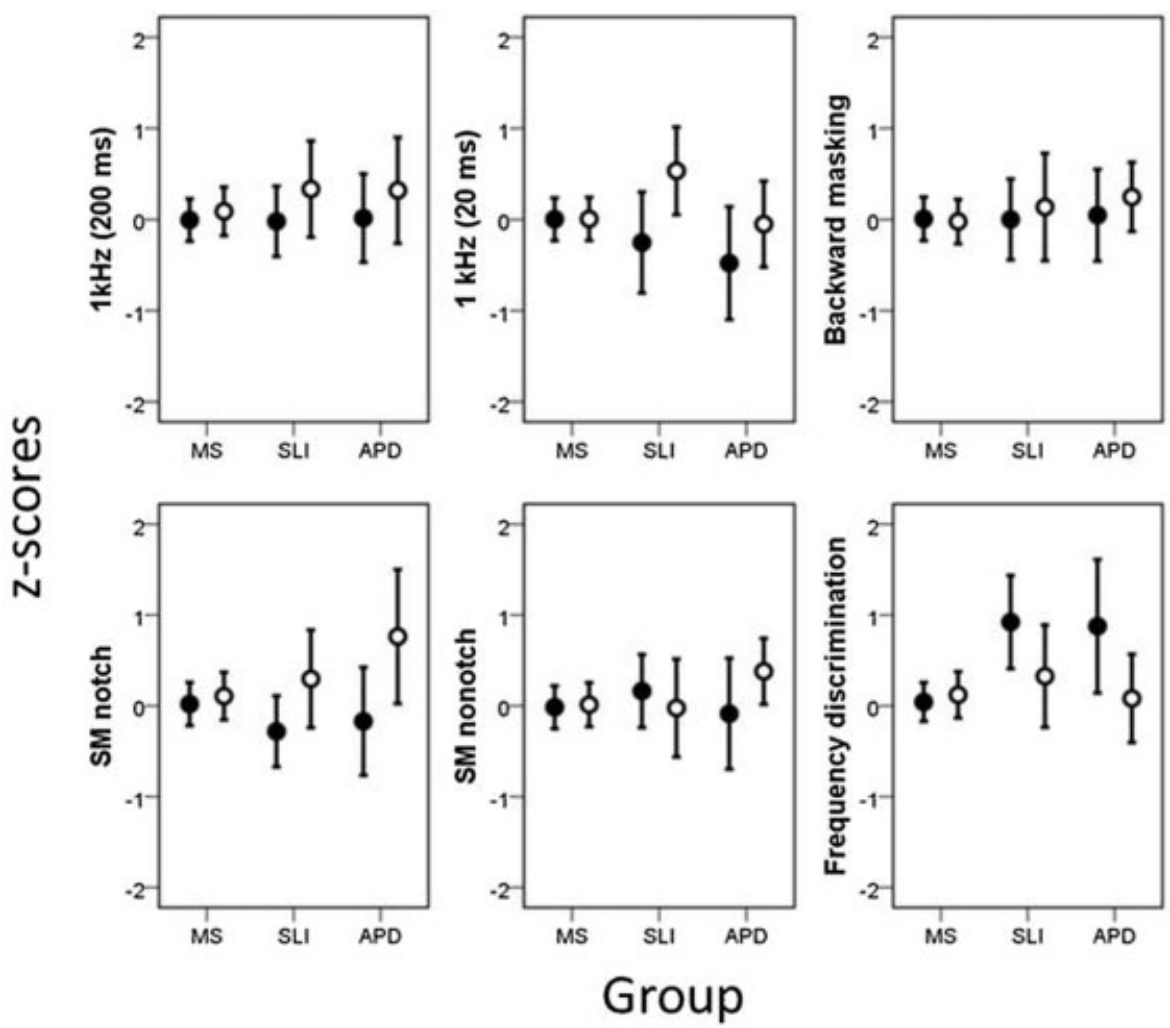

Figure 3 Mean and 95\% confidence intervals for auditory processing variability measures, expressed in agestandardized z-scores for standard deviation (open circles) and intertrack threshold difference (solid circles) between the mainstream school (MS), specific language impairment (SLI), and auditory processing disorder (APD) groups. Abbreviation: SM, simultaneous masking. 
no significant difference between the SLI and APD groups.

The contribution of the three nonsensory factors (intrinsic attention, age, and NVIQ) to a model of multiple regression on auditory processing threshold (not standardized for age) with intrinsic attention entered first, followed by age and NVIQ, is shown in Table 4. For the MS group, adding age to the model accounted for a much larger proportion of threshold variance (between 10 and 46\%) than adding SD (4 to 19\%). It was notable that adding NVIQ to the model did not explain any additional variance after SD and age were accounted for. Thus, for the MS children, age made the largest contribution to auditory processing threshold. This was not the case for the SLI/APD children. For those groups, the variance accounted for by intrinsic attention was broadly similar to the

\begin{tabular}{|c|c|c|c|}
\hline Test & SD & $\begin{array}{c}R^{2} \\
\text { SD }+ \text { Age }\end{array}$ & SD + Age + NVIO \\
\hline \multicolumn{4}{|l|}{ MS } \\
\hline $1 k 200$ & $0.17^{\ddagger}$ & $0.28^{\ddagger}$ & $0.29^{\ddagger}$ \\
\hline $1 \mathrm{k} 20$ & $0.12^{+}$ & $0.46^{\ddagger}$ & $0.44^{\ddagger}$ \\
\hline BM & $0.12^{\ddagger}$ & $0.40^{\ddagger}$ & $0.39^{\ddagger}$ \\
\hline SMO & $0.11^{\dagger}$ & $0.32^{\ddagger}$ & $0.35^{\ddagger}$ \\
\hline SMN & $0.19^{\ddagger}$ & $0.47^{\ddagger}$ & $0.46^{\ddagger}$ \\
\hline FD & 0.04 & 0.10 & 0.16 \\
\hline \multicolumn{4}{|l|}{ SLI/APD } \\
\hline 1 k200 & $0.35^{\ddagger}$ & $0.34^{\ddagger}$ & $0.35^{\ddagger}$ \\
\hline $1 \mathrm{k} 20$ & 0.05 & 0.06 & 0.11 \\
\hline BM & $0.17^{*}$ & $0.19^{*}$ & $0.27^{*}$ \\
\hline SMO & $0.17^{*}$ & $0.29^{\dagger}$ & $0.33^{\dagger}$ \\
\hline SMN & 0.07 & 0.07 & 0.10 \\
\hline FD & $0.15^{*}$ & 0.16 & 0.17 \\
\hline
\end{tabular}

The contributions of adding age, and then $\mathrm{NVIQ}$, to the multiple regression model are shown. $1 \mathrm{k} 20$, tone duration 20 milliseconds; 1k200, tone duration 200 milliseconds; ANOVA, analysis of variance; APD, auditory processing disorder; AP, auditory processing; $B M$, backward masking; FD, frequency discrimination; $F R$, frequency resolution; MANOVA, multivariate analysis of variance; MS, mainstream school; NS, not significant; NVIQ, nonverbal IQ; SD, standard deviation; SLI, specific language impairment; SM0, no-notch condition; SMN, notch condition; $\mathrm{Tl}$, temporal integration.

${ }^{*} p \leq 0.05$.

$+p \leq 0.01$

${ }^{\ddagger} p \leq 0.001$.
MS group, but age accounted for considerably less variance in the clinical groups. After accounting for SD and age, only BM showed a significant additional contribution of NVIQ.

\section{DISCUSSION}

The two clinical groups (SLI and APD) consistently underperformed compared to the MS group on thresholds for most of the auditory processing tests. Furthermore, there was no significant difference in thresholds for any of the auditory processing tests between the SLI and APD groups. Several other studies also have shown poorer auditory processing abilities, compared with TD children, in children from clinical groups including SLI, ${ }^{9,13,17,70}$ dyslexia, ${ }^{23,71,72}$ and APD. ${ }^{50,73}$ In the present study, when NVIQ was accounted for, the group differences between the MS and the clinical groups disappeared for all auditory processing tests except BM. This suggests that the agestandardized auditory processing deficits seen in the clinical groups were not specific to the auditory stimuli alone and that perception of the auditory stimuli also was influenced by nonsensory cognitive factors. For the children with SLI and APD studied here, this was further evidenced by some significant associations between auditory processing thresholds and NVIQ. These results are consistent with reanalyzed data from several studies in children and adults with LLI, ${ }^{46}$ where accounting for NVIQ significantly reduced or even abolished the previously reported relationship between auditory processing and language and literacy measures.

The poorer performance in the individual auditory processing thresholds shown for the SLI and APD groups compared with the MS group in the present study are broadly consistent with results reported by Moore et al. ${ }^{4}$ This large population study of normally hearing children showed that those children with poorer listening, communication, and speech intelligibility (i.e., the presenting symptoms of APD) also had poorer auditory processing thresholds for the individual tests. However, Moore et al also showed that the presenting symptoms of APD were best predicted by intrinsic attention, indexed by within-response 
variability, and by "cognition" (a composite measure including NVIQ, memory, reading, and language). ${ }^{4}$ Although our previously published data from the same participant sample presented in this article showed highly significant differences between the MS and clinical groups for communication (CCC-2), listening (CHAPPS), and cognition (NVIQ and memory), ${ }^{49}$ there were generally no differences between the MS, SLI, and APD groups for intrinsic attention. Thus, there was little evidence of the findings of Moore et al on the relationship between intrinsic attention and clinical presenting symptoms of APD in the present study. ${ }^{4}$ Interestingly, however, intrinsic attention contributed more to the auditory processing thresholds than age in the SLI and APD groups, whereas age contributed more to auditory processing thresholds than intrinsic attention in the MS group compared with the clinical groups. Therefore it may be inferred that intrinsic attention plays a relatively larger role in auditory task performance in children with language or listening deficits than in TD children.

Very few studies have assessed intrinsic attention in either TD or clinical samples. Dawes et al showed a general lack of correlation between a similar attention measure (SD of the track reversals) for FM detection tasks and thresholds, but intrinsic attention was not reported separately for the TD, APD, or dyslexic groups in that study. ${ }^{23}$ However, Sutcliffe et al showed that an intrinsic attention measure for a frequency discrimination task (SD for track reversals) and a between-tracks measure (threshold variance estimates for three tracks) differed significantly between children with ADHD and a control group of TD children. ${ }^{31}$ Greater variability in the SD measure was shown in the ADHD group, irrespective of whether the children were taking prescribed stimulant medication or not. However, differences in medication were shown to affect performance on only the between-tracks measure, which showed larger deterioration in performance when subjects were off medication compared with on medication. Sutcliffe et al offered an explanation that the FD task, ${ }^{31}$ based on relatively short-duration signals, tapped into temporal synchronization of attention in a way similar to symptoms described in children with ADHD. ${ }^{74}$ They noted, however, that this temporal attention mechanism was different than the temporal processing hypothesis of Tallal and Piercy that proposes an inability to detect differences between two sounds due to poor temporal resolution in the auditory system. $^{12}$

That none of the experimental studies cited showed a consistent difference between clinical groups and TD controls in intrinsic attention indexed by the measures used here (SD in track responses or track reversals) may be because these intrinsic attention measures are not sensitive enough to show a difference in small participant samples. Importantly, for clinical evaluations, these measures are not diagnostically useful at the individual level. The population study that did show intrinsic attention was a factor in APD presenting symptoms included 1469 children and analyzed 18 measures of response variability. ${ }^{4}$ Thus, that study had the power to show an effect that explained a relatively small amount of the variance, between 5 and $9 \%$, for each of the speech intelligibility, communication, and listening measures. Alternately, the remote test center design of the Moore et al study may not have led to as much control of attention as is possible in laboratory-based studies. Nevertheless, there is growing support for the concept of attention as an explanation for poor speech intelligibility, communication, and listening abilities. ${ }^{4,10,73,75}$ A final note of caution, however, is that we do not understand the direction of causality between auditory processing and cognitive processing. It remains a possibility that some form of sensory impairment, not detected by an audiogram, can give an impression of cognitive impairment. Returning to Spearman in $1904,{ }^{42}$ it has long been proposed that sensory function may determine intelligence, so the observed contributions of NVIQ to auditory processing and language may themselves be a reflection of an effect of hearing on NVIQ.

\section{ACKNOWLEDGMENTS}

This paper presents independent research funded by the Medical Research Council, 
Nottingham University Hospitals NHS Trust and the National Institute for Health Research (NIHR) Biomedical Research Unit Program. The views expressed are those of the authors and not necessarily those of the National Health Service, the NIHR, or the Department of Health.

\section{REFERENCES}

1. American Academy of Audiology. Diagnosis, Treatment and Management of Children and Adults with Central Auditory Processing Disorder. 2010. Available at: http://www.audiology.org/resources/documentlibrary/documents/capd\%20guidelines\%2082010.pdf. Accessed November 1, 2013

2. British Society of Audiology. Position Statement: Auditory Processing Disorder (APD). 2011. Available at: http://www.thebsa.org.uk/docs/docsfromold/ BSA_APD_PositionPaper_31March11_FINAL. pdf. Accessed November 1, 2013

3. British Society of Audiology. Auditory Processing Disorder Steering Committee Interim Position Statement on APD. Reading: British Society of Audiology

4. Moore DR, Ferguson MA, Edmondson-Jones AM, Ratib S, Riley A. Nature of auditory processing disorder in children. Pediatrics 2010;126(2): e382-e390

5. Schacter D, Gilbert D, Wegner D. Psychology. 2nd ed. New York, NY: Worth; 2011

6. Bishop DV, Carlyon RP, Deeks JM, Bishop SJ. Auditory temporal processing impairment: neither necessary nor sufficient for causing language impairment in children. J Speech Lang Hear Res 1999;42(6):1295-1310

7. Rosen S, Manganari E. Is there a relationship between speech and nonspeech auditory processing in children with dyslexia? J Speech Lang Hear Res 2001;44(4):720-736

8. Amitay S, Ahissar M, Nelken I. Auditory processing deficits in reading disabled adults. J Assoc Res Otolaryngol 2002;3(3):302-320

9. Rosen S, Adlard A, van der Lely HK. Backward and simultaneous masking in children with grammatical specific language impairment: no simple link between auditory and language abilities. J Speech Lang Hear Res 2009;52(2):396-411

10. Dawes P, Bishop D. Auditory processing disorder in relation to developmental disorders of language, communication and attention: a review and critique. Int J Lang Commun Disord 2009;44(4): 440-465
11. Witton C. Childhood auditory processing disorder as a developmental disorder: the case for a multiprofessional approach to diagnosis and management. Int J Audiol 2010;49(2):83-87

12. Tallal P, Piercy M. Defects of non-verbal auditory perception in children with developmental aphasia. Nature 1973;241(5390):468-469

13. Wright BA, Buonomano DV, Mahncke HW, Merzenich MM. Learning and generalization of auditory temporal-interval discrimination in humans. J Neurosci 1997;17(10):3956-3963

14. Talcott JB, Witton C, Hebb GS, et al. On the relationship between dynamic visual and auditory processing and literacy skills; results from a large primary-school study. Dyslexia 2002;8(4): 204-225

15. Witton C, Stein JF, Stoodley CJ, Rosner BS, Talcott JB. Separate influences of acoustic AM and FM sensitivity on the phonological decoding skills of impaired and normal readers. J Cogn Neurosci 2002;14(6):866-874

16. Cestnick L, Jerger J. Auditory temporal processing and lexical/nonlexical reading in developmental dyslexics. J Am Acad Audiol 2000;11(9):501-513

17. McArthur GM, Bishop DV. Auditory perceptual processing in people with reading and oral language impairments: current issues and recommendations. Dyslexia 2001;7(3):150-170

18. Bishop DV, McArthur GM. Individual differences in auditory processing in specific language impairment: a follow-up study using event-related potentials and behavioural thresholds. Cortex 2005;41(3):327-341

19. Bailey PJ, Snowling MJ. Auditory processing and the development of language and literacy. Br Med Bull 2002;63(1):135-146

20. King WM, Lombardino LJ, Crandell CC, Leonard $\mathrm{CM}$. Comorbid auditory processing disorder in developmental dyslexia. Ear Hear 2003;24(5): 448-456

21. Marler JA, Champlin CA, Gillam RB. Auditory memory for backward masking signals in children with language impairment. Psychophysiology 2002;39(6):767-780

22. Ramus F, Rosen S, Dakin SC, et al. Theories of developmental dyslexia: insights from a multiple case study of dyslexic adults. Brain 2003;126(Pt 4): 841-865

23. Dawes $P$, Sirimanna T, Burton M, Vanniasegaram I, Tweedy F, Bishop D. Temporal auditory and visual motion processing of children diagnosed with auditory processing disorder and dyslexia. Ear Hear 2009;30(6):1-11

24. Hill PR, Hartley DE, Glasberg BR, Moore BC, Moore DR. Auditory processing efficiency and temporal resolution in children and adults. J Speech Lang Hear Res 2004;47(5):1022-1029 
25. Nozza RJ. Estimating the contribution of nonsensory factors to infant-adult differences in behavioral thresholds. Hear Res 1995;91(1-2):72-78

26. Dawes P, Bishop DVM. Maturation of visual and auditory temporal processing in school-aged children. J Speech Lang Hear Res 2008;51(4):1002-1015

27. Hartley DE, Wright BA, Hogan SC, Moore DR. Age-related improvements in auditory backward and simultaneous masking in 6- to 10-year-old children. J Speech Lang Hear Res 2000;43(6): 1402-1415

28. Buss E, Hall JW, Grose JH, Dev MB. A comparison of threshold estimation methods in children 611 years of age. J Acoust Soc Am 2001;109(2): $727-731$

29. Wightman FL, Allen P. Individual differences in auditory capability among preschool children. In: Werner LA, Rubel E, eds. Developmental Psychoacoustics. Washington DC: American Psychological Association; 1992:113-133

30. Allen P, Wightman F, Kistler D, Dolan T. Frequency resolution in children. J Speech Hear Res 1989;32(2):317-322

31. Sutcliffe PA, Bishop DV, Houghton S, Taylor M. Effect of attentional state on frequency discrimination: a comparison of children with ADHD on and off medication. J Speech Lang Hear Res 2006; 49(5):1072-1084

32. Hartley DE, Moore DR. Auditory processing efficiency deficits in children with developmental language impairments. J Acoust Soc Am 2002; 112(6):2962-2966

33. Moore DR, Cowan JA, Riley A, EdmondsonJones AM, Ferguson MA. Development of auditory processing in 6- to 11-yr-old children. Ear Hear 2011;32(3):269-285

34. Wightman F, Allen P, Dolan T, Kistler D, Jamieson D. Temporal resolution in children. Child Dev 1989;60(3):611-624

35. Roach NW, Edwards VT, Hogben JH. The tale is in the tail: an alternative hypothesis for psychophysical performance variability in dyslexia. Perception 2004;33(7):817-830

36. Moore DR, Ferguson MA, Halliday LF, Riley A. Frequency discrimination in children: perception, learning and attention. Hear Res 2008;238(1-2): 147-154

37. Manly T, Anderson V, Nimmo-Smith I, Turner A, Watson P, Robertson IH. The differential assessment of children's attention: the Test of Everyday Attention for Children (TEA-Ch), normative sample and ADHD performance. J Child Psychol Psychiatry 2001;42(8):1065-1081

38. Smoski WJ, Brunt MA, Tannahill JC. Listening characteristics of children with central auditory processing disorders. Lang Speech Hear Serv Sch 1992;23(2):145-152
39. Bishop D. Children's Communication Checklist (CCC-2). 2nd ed. London, UK: The Psychological Corporation; 2003

40. Deary IJ. Auditory inspection time and intelligence: what is the direction of causation? Dev Psychol 1995;31(2):237-250

41. Raz N, Moberg PJ, Millman D. Effects of age and age-related differences in auditory information processing on fluid and crystallized intelligence. Pers Individ Dif 1990;11(11):1147-1152

42. Spearman C. "General intelligence," objectively determined and measured. Am J Psychol 1904; 15(2):201-292

43. Witton C, Talcott JB, Hansen PC, et al. Sensitivity to dynamic auditory and visual stimuli predicts nonword reading ability in both dyslexic and normal readers. Curr Biol 1998;8(14):791-797

44. Goswami U, Thomson J, Richardson U, et al. Amplitude envelope onsets and developmental dyslexia: a new hypothesis. Proc Natl Acad Sci U S A 2002;99(16):10911-10916

45. Ahissar M, Protopapas A, Reid M, Merzenich MM. Auditory processing parallels reading abilities in adults. Proc Natl Acad Sci U S A 2000;97(12): 6832-6837

46. Rosen S. Auditory processing in dyslexia and specific language impairment: is there a deficit? What is its nature? Does it explain anything?. J Phonetics 2003;31(3):509-527

47. Hulslander J, Talcott J, Witton C, et al. Sensory processing, reading, IQ and attention. J Exp Child Psychol 2004;88(3):274-295

48. Miller CA, Wagstaff DA. Behavioural profiles associated with auditory processing disorder and specific language impairment. J Commun Dis 2011;44:745-763

49. Ferguson MA, Hall RL, Riley A, Moore DR. Communication, listening, speech and cognition in children diagnosed with auditory processing disorder (APD) or specific language impairment (SLI). J Speech Lang Hear Res 2011; 54:211-227

50. Rosen S, Cohen M, Vanniasegaram I. Auditory and cognitive abilities of children suspected of auditory processing disorder (APD). Int J Pediatr Otorhinolaryngol 2010;74(6):594-600

51. Kiessling J, Pichora-Fuller MK, Gatehouse S, et al. Candidature for and delivery of audiological services: special needs of older people. Int J Audiol 2003;42(2, Suppl 2):S92-S101

52. Wingfield A, Tun PA, McCoy SL. Hearing loss in older adulthood what it is and how it interacts with cognitive performance. Curr Dir Psychol Sci 2005; 14(3):144-148

53. Arlinger S, Lunner T, Lyxell B, Pichora-Fuller MK. The emergence of cognitive hearing science. Scand J Psychol 2009;50(5):371-384 
54. Moore DR. Listening difficulties in children: bottom-up and top-down contributions. J Commun Disord 2012;45(6):411-418

55. Leonard LB. Children with Specific Language Impairment. Cambridge, MA: MIT Press; 1998

56. Bamiou DE, Luxon LM. Auditory processing disorders. BMJ 2008;337:a2080

57. Moore DR, Rosen S, Bamiou D-E, Campbell NG, Sirimanna T. Evolving concepts of developmental auditory processing disorder (APD): a British Society of Audiology APD special interest group “white paper." Int J Audiol 2013;52(1):3-13

58. Dillon H, Cameron S, Glyde H, Wilson W, Tomlin D. An opinion on the assessment of people who may have an auditory processing disorder. J Am Acad Audiol 2012;23(2):97-105

59. Jutras B, Loubert M, Dupuis JL, Marcoux C, Dumont V, Baril M. Applicability of central auditory processing disorder models. Am J Audiol 2007; 16(2):100-106

60. Wilson WJ, Arnott W. Using different criteria to diagnose (central) auditory processing disorder: how big a difference does it make? J Speech Lang Hear Res 2013;56(1):63-70

61. Moore DR. Auditory processing disorder (APD): definition, diagnosis, neural basis, and intervention. Audiol Med 2006;4(1):4-11

62. Dawes P, Bishop DV, Sirimanna T, Bamiou DE. Profile and aetiology of children diagnosed with auditory processing disorder (APD). Int J Pediatr Otorhinolaryngol 2008;72(4):483-489

63. Hind S. Survey of care pathway for auditory processing disorder. Audiol Med 2006;4(1):12-24

64. Bamiou DE, Musiek FE, Luxon LM. Aetiology and clinical presentations of auditory processing disorders-a review. Arch Dis Child 2001;85(5): 361-365

65. ASHA. Central Auditory Processing Disorders. American Speech Language Hearing Association;
2005. Available at: http://www.asha.org/policy/ TR2005-00043/. Accessed November 1, 2013

66. Levitt H. Transformed up-down methods in psychoacoustics. J Acoust Soc Am 1971;49(2):2, 467

67. Wechsler D. Wechsler Abbreviated Scale of Intelligence. Psychological Corporation. New York, NY: Harcourt Brace \& Company; 1999

68. Chermak GD, Musiek FE, Craig CH. Central Auditory Processing Disorders: New Perspectives. San Diego, CA: Singular Publishing Group; 1997

69. Hind SE, Haines-Bazrafshan R, Benton CL, Brassington W, Towle B, Moore DR. Prevalence of clinical referrals having hearing thresholds within normal limits. Int J Audiol 2011;50(10):708-716

70. Corriveau K, Pasquini E, Goswami U. Basic auditory processing skills and specific language impairment: a new look at an old hypothesis. J Speech Lang Hear Res 2007;50(3):647-666

71. Heath SM, Hogben JH, Clark CD. Auditory temporal processing in disabled readers with and without oral language delay. J Child Psychol Psychiatry 1999;40(4):637-647

72. McArthur GM, Hogben JH. Auditory backward recognition masking in children with a specific language impairment and children with a specific reading disability. J Acoust Soc Am 2001;109(3): 1092-1100

73. Vanniasegaram I, Cohen M, Rosen S. Evaluation of selected auditory tests in school-age children suspected of auditory processing disorders. Ear Hear 2004;25(6):586-597

74. Castellanos FX, Tannock R. Neuroscience of attention-deficit/hyperactivity disorder: the search for endophenotypes. Nat Rev Neurosci 2002;3(8): 617-628

75. Loo JH, Bamiou DE, Rosen S. The impacts of language background and language-related disorders in auditory processing assessment. J Speech Lang Hear Res 2013;56(1):1-12 